Cite this: RSC Adv., 2014, 4, 29847

\title{
Supramolecular assemblies of semiconductor quantum dots and a bis(bipyridinium) derivative: luminescence quenching and aggregation phenomena $\uparrow$
}

\author{
Marek Oszajca, ${ }^{\text {ab }}$ Christophe Lincheneau, ${ }^{a}$ Matteo Amelia, ${ }^{a}$ Massimo Baroncini, ${ }^{a}$ \\ Serena Silvi, ${ }^{a}$ Konrad Szaciłowski ${ }^{\text {bc }}$ and Alberto Credi ${ }^{* a}$
}

\begin{abstract}
We have synthesized CdSe and CdSe-ZnS core-shell luminescent nanocrystal quantum dots and studied their interaction with a ditopic bis(bipyridinium) compound in solution. The latter strongly quenches the luminescence of the quantum dots by a static mechanism, indicating that the nanocrystal and molecular components undergo association in the ground state. Photoexcitation of these inorganic-organic hybrids causes an electron-transfer process from the conduction band of the nanocrystal to the LUMO of the molecule. The ability of the bipyridinium-type species to trigger association of the quantum dots is evidenced by spectrofluorimetric titrations and DLS measurements in solution, and confirmed by TEM experiments on surfaces. The quantum dot-molecule complexes can be disassembled in solution by addition of a calixarene host capable of encapsulating the bipyridinium units of the molecular connector. Our results demonstrate that supramolecular chemistry offers convenient ways to control the aggregation of semiconductor nanocrystals, a crucial task for the generation of nanostructured arrays with well defined properties.
\end{abstract}

Received 10th April 2014

Accepted 20th June 2014

DOI: $10.1039 / c 4 r a 03259 d$

www.rsc.org/advances synthesis of derivatives in which the molecule of interest is connected to a ligand moiety for the QD surface, and the optimization of exchange procedures in order to replace the native ligands of the nanocrystal with the functional ones. Several recent reports, however, have shown ${ }^{7,8}$ that bare semiconductor nanocrystals and functional molecular components can be selfassembled in an efficient manner taking advantage of supramolecular interactions in solution. This approach is advantageous because (i) no exchange of the capping ligands of the quantum dots is required, (ii) the molecular components do not need to have a surface docking unit, and (iii) the association process, relying on non-covalent interactions, is reversible under appropriate conditions.

In a previous investigation ${ }^{9}$ we showed that CdSe-ZnS coreshell QDs form remarkably stable supramolecular complexes with $1,1^{\prime}$-dialkyl-4,4'-bipyridinium salts in nonpolar solvents, most likely owing to a combination of van der Waals forces between the guest and the surface-bound molecular monolayer, and solvophobic effects experienced by the positively charged guest. These complexes may also be stabilized by electrostatic interactions between the bipyridinium units and the negatively charged ZnS surface. ${ }^{10}$ The formation of these complexes is signalled by the decrease of the luminescence intensity of the nanocrystals, which is quenched because of a photoinduced electron-transfer process from the QD conduction band to the LUMO of the nearby bipyridinium unit., ${ }^{9,11,12}$ The original

\footnotetext{
aPhotochemical Nanosciences Laboratory, Dipartimento di Chimica "G. Ciamician", Università di Bologna, Via Selmi 2, 40126 Bologna, Italy. E-mail: alberto.credi@ unibo.it; Fax: +39051 2099456; Tel: +39 0512099540

${ }^{b}$ Faculty of Chemistry, Jagiellonian University, Ingardena 3, 30-060 Krakow, Poland ${ }^{c}$ Faculty of Non-Ferrous Metals, AGH University of Science and Technology, al. Mickiewicza 30, 30-060 Kraków, Poland

$\dagger$ Electronic supplementary information (ESI) available: UV-visible absorption spectra, luminescence spectra and lifetimes, and TEM images. See DOI: $10.1039 / \mathrm{c} 4 \mathrm{ra03259d}$
} 
luminescence intensity of the nanoparticles could be quantitatively restored upon addition of a calix[6]arene host able to compete with the QDs for the bipyridinium quenchers. ${ }^{9}$

On the basis of these results we designed and synthesized a new bis(bipyridinium) derivative $\mathbf{1}^{\mathbf{4}+}$ (Chart 1 ), composed of two $4,4^{\prime}$-bipyridinium units linked together by means of a $p$-xylylene spacer and bearing decyl side chains. We then investigated the association of $\mathbf{1}^{\mathbf{4}}$ with either CdSe or CdSe-ZnS core-shell quantum dots in organic solution, using absorption and luminescence spectroscopy, dynamic light scattering, and transmission electron microscopy. The complexation of the bipyridinium units of $\mathbf{1}^{\mathbf{4 +}}$ by the calix[6]arene host 2 was also investigated and successively exploited to cause the detachment of $\mathbf{1}^{\mathbf{4}+}$ from the nanocrystals by competitive binding.

The general motivation of this work is to identify simple and efficient strategies for assembling quantum dot and molecular components, and to increase our knowledge of nanocrystalmolecule interactions. More specifically, the present study is aimed at understanding whether the ditopic structure of the $\mathbf{1}^{\mathbf{4 +}}$ guest can be exploited to induce the aggregation of the nanocrystals in solution, and if such a phenomenon is chemically reversible. Using supramolecular complexation to control aggregation of semiconductor nanocrystals ${ }^{\mathbf{1 3}}$ is a promising route for the construction of colloidal superparticles ${ }^{\mathbf{1 4}}$ and ordered two- and three-dimensional nanocrystal arrays (superlattices and artificial solids ${ }^{15}$ that could exhibit useful chemical and optoelectronic properties. ${ }^{16}$

\section{Experimental}

\section{Materials and methods}

Cadmium oxide (CdO, 99.99\%), 1-hexadecylamine (HDA, 98\%), tri- $n$-octylphosphine oxide (TOPO, 99\%), 1- $n$-octadecene (ODE, 90\%), oleic acid (OA, 99\%) and $n$-octadecylamine (ODA, 99\%) were purchased from Aldrich; elemental selenium (99.999\%,

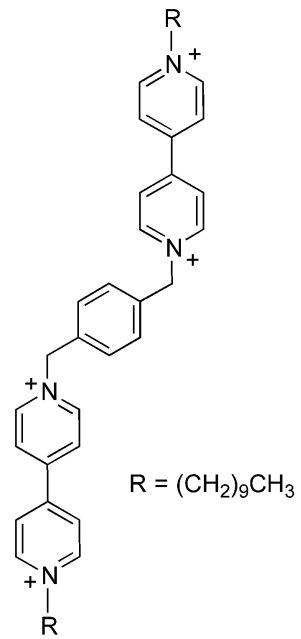

$1^{4+}$

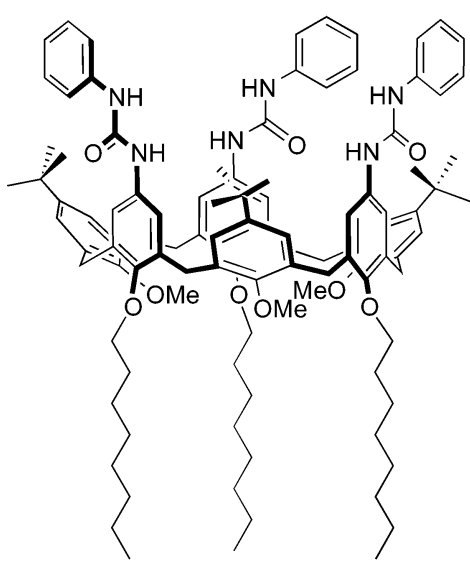

2
Chart 1 Structural formulas of the investigated molecular components.
200 mesh), and tri- $n$-butylphosphine (97\%) were from Alfa Aesar. 1,4-Bis(1-decyl-4,4'-bipyridinium-1'-methyl)benzene tetrachloride $(\mathbf{1} \cdot 4 \mathrm{Cl})$ was synthesized according to a previously published procedure. ${ }^{17}$ Commercially available compounds were reagent grade quality and were used without further purification. The tris( $N$-phenylureido)calix[6] arene 2 was a gift from Profs A. Arduini and A. Secchi, Università di Parma, and was available from previous investigations..${ }^{9,18}{ }^{1} \mathrm{H}$ And ${ }^{13} \mathrm{C} \mathrm{NMR}$ spectra were recorded at $295 \mathrm{~K}$ with a Varian Mercury 400 spectrometer with the deuterated solvent as the lock and the residual solvent as the internal standard. All chemical shifts are quoted using the $\delta$ scale, and all coupling constants $(J)$ are expressed in Hertz (Hz). Melting points were determined using a Büchi 510 series apparatus and are uncorrected. Air equilibrated chloroform and acetonitrile (Merck Uvasol ${ }^{\mathrm{TM}}$ ) were used as the solvents for the spectroscopic measurements.

Absorption and luminescence spectra were recorded in either air-equilibrated or deoxygenated (argon purged) solutions with a Perkin Elmer Lambda 45 spectrophotometer and a Perkin-Elmer LS50B spectrofluorimeter equipped with a Hamamatsu R928 phototube, respectively. Luminescence lifetimes were measured with an Edinburgh Instruments FLS920 time-correlated single-photon counting spectrofluorimeter, exciting the sample at $405 \mathrm{~nm}$ with a pulsed laser.

Titration experiments were carried out by adding small aliquots of a concentrated stock solution of $\mathbf{1}^{\mathbf{4 +}}$ to a known volume of a dilute nanocrystal solution. In all instances the absorbance at the excitation wavelength was small enough to avoid inner filter effects and take advantage of the linear relationship between absorbed and emitted light. ${ }^{19}$ The experimental errors are $\pm 1 \mathrm{~nm}$ for the wavelength values, $\pm 10 \%$ for the luminescence intensities and lifetimes, and $\pm 20 \%$ for the stability constants.

The nanoparticle size distribution was determined by transmission electron microscopy (TEM) and dynamic light scattering (DLS) measurements. TEM experiments were carried out with a Philips CM 100 transmission electron microscope operating at $80 \mathrm{kV}$, while HRTEM measurements were carried out with a FEI Tecnai Osiris S/TEM system operating at $200 \mathrm{kV}$. A drop of the nanocrystal solution diluted with hexane was deposited on a 400 mesh copper coated with formvar support grid (TAAB Ltd.), which was then dried up under vacuum to remove any solvent trace. DLS experiments were performed with a Malvern Nano ZS instrument equipped with a $633 \mathrm{~nm}$ laser diode. The measurements were carried out at $20{ }^{\circ} \mathrm{C}$ in airequilibrated solutions placed in $1 \mathrm{~cm}$ quartz cuvettes. The samples were filtered twice through $0.1 \mu \mathrm{m}$ PTFE membrane filters prior to the measurements. The resulting size is the average of at least 20 independent experiments, and the standard deviation is taken as the experimental error.

\section{Synthesis}

1,4-Bis(1-decyl-4,4'-bipyridinium-1'-methyl)benzene hexafluorophosphate $\left(\mathbf{1} \cdot \mathbf{4 P F}_{\mathbf{6}}\right) \cdot 1,4$-Bis(1-decyl-4,4'-bipyridinium-1' methyl)benzene tetrachloride ${ }^{17}$ was dissolved in hot water and to the clear mixture a saturated aqueous solution of $\mathrm{NH}_{4} \mathrm{PF}_{6}$ was 
added dropwise. The white precipitate was filtered, washed with water and THF, and dried in vacuo overnight to yield the title compound in quantitative yield as a pale yellow powder. M.p. = $>180{ }^{\circ} \mathrm{C}$ (dec.); ${ }^{1} \mathrm{H}-\mathrm{NMR}\left(400 \mathrm{MHz}\right.$, acetone- $\left.\mathrm{d}_{6}\right): \delta=9.48(\mathrm{~d}, J=7$ $\mathrm{Hz}, 4 \mathrm{H}), 9.43$ (d, $J=7 \mathrm{~Hz}, 4 \mathrm{H}), 8.30(\mathrm{~m}, 8 \mathrm{H}), 7.80(\mathrm{~s}, 4 \mathrm{H}), 6.23(\mathrm{~s}$, $4 \mathrm{H}), 4.95(\mathrm{~m}, 4 \mathrm{H}), 2.09(\mathrm{~m}, 4 \mathrm{H}), 1.25-1.52(\mathrm{~m}, 28 \mathrm{H}), 0.89(\mathrm{~m}$, $6 \mathrm{H}) ;{ }^{13} \mathrm{C}-\mathrm{NMR}$ (101 MHz, acetone- $\left.\mathrm{d}_{6}\right): \delta=155.37,150.25$, $144.40,143.87,133.32$, 130.65, 128.54, 128.42, 65.01, 62.43, $33.12,32.69,30.82,30.78,30.57,30.42,27.43,24.12,15.62 . \mathrm{MS}$ (ESI): $m / z 1133.9\left[\mathrm{M}-\mathrm{PF}_{6}\right]^{+}$.

CdSe nanocrystals. The synthesis of CdSe core QDs was achieved following the procedures developed by Peng and coworkers, ${ }^{20}$ with minor modifications. ${ }^{21}$ In brief, the nucleation of the QDs was obtained by reacting the cadmium(II) precursor with elemental selenium coordinated by TBP at $270{ }^{\circ} \mathrm{C}$ in a solvent with a high boiling point. ODE was used as the solvent, and TOPO and HDA were employed as the surface ligands. In the growth phase, the temperature was lowered to $250{ }^{\circ} \mathrm{C}$. In a typical synthetic batch, $0.20 \mathrm{mmol}$ of $\mathrm{CdO}$ and 0.80 mmol of OA were heated at $170{ }^{\circ} \mathrm{C}$ in a three-neck flask connected to a Schlenk line whilst stirring and under an argon flow until the reaction was completed (the solution turned from reddish to clear). After cooling the mixture to room temperature, $0.5 \mathrm{~g}$ of TOPO, $1.5 \mathrm{~g}$ of HDA and $2.0 \mathrm{~g}$ of ODE were added, and the mixture was heated to $270{ }^{\circ} \mathrm{C}$. At this temperature a swift injection of a room-temperature Se stock solution (2.0 mmol Se, $0.45 \mathrm{~g}$ TBP and $1.4 \mathrm{~g}$ of ODE, prepared in a glove box and transferred by syringe) was performed. The temperature was set at $250{ }^{\circ} \mathrm{C}$ immediately after the injection in order to allow the nanocrystals to grow. After cooling to room temperature the QDs were purified by 3 extraction cycles with a $1: 1$ methanol-hexane mixture.

CdSe-ZnS core-shell nanocrystals. CdSe-ZnS nanocrystals were prepared by overcoating a CdSe core with a $\mathrm{ZnS}$ shell using the SILAR procedure. ${ }^{20}$ In brief, $\mathrm{ZnO}(0.4 \mathrm{mmol})$ was dissolved in a mixture of ODA $(1 \mathrm{~mL})$ and $\mathrm{ODE}(9 \mathrm{~mL})$ at $250^{\circ} \mathrm{C}$, and $\mathrm{S}(0.4$ $\mathrm{mmol})$ was dissolved in ODE $(10 \mathrm{~mL})$ at $200{ }^{\circ} \mathrm{C}$. The precalculated amounts of $\mathrm{Zn}$ cations and $\mathrm{S}$ anions were heteroepitaxially deposited through an air-free standard syringe procedure onto the previously synthesized CdSe nanocrystals. Four layers of ZnS were deposited on top of the core QDs. As the shell thickness for a full monolayer of $\mathrm{ZnS}$ is $0.31 \mathrm{~nm},{ }^{22}$ the QD diameter increases of $2.5 \mathrm{~nm}$ after the deposition of the four monolayers.

\section{Results and discussion}

\section{CdSe nanocrystals}

The investigated CdSe core nanocrystals in $\mathrm{CHCl}_{3}$ exhibit a broad UV-visible absorption and the characteristic low energy exciton peak $\left(\lambda_{\max }=560 \mathrm{~nm}\right)$ arising from quantum confinement of the charge carriers. ${ }^{1-3}$ The diameter of the nanocrystals, determined from the position of their low-energy absorption peak according to a published procedure, ${ }^{23}$ resulted to be 3.3 $\mathrm{nm}$, while the molar absorption coefficient of the exciton peak is $\varepsilon_{\max }=134000 \mathrm{M}^{-1} \mathrm{~cm}^{-1}$. TEM Images revealed that the particles are spherical and well monodispersed, as expected from the applied synthetic methodology. The luminescence spectrum of the QDs in air equilibrated $\mathrm{CHCl}_{3}$ at room temperature shows the intense and narrow luminescence band $\left(\lambda_{\max }=578 \mathrm{~nm}\right.$, fwhm $\left.=30 \mathrm{~nm}\right)$ typical of radiative exciton recombination. Such an emission exhibit a decay that could be satisfactorily fitted with a double exponential function, yielding an average lifetime of $33 \mathrm{~ns} . \dagger$

Because the bis(bipyridinium) compound $\mathbf{1} \cdot 4 \mathrm{PF}_{6}$ is insoluble in $\mathrm{CHCl}_{3}$, its properties were examined in $\mathrm{CHCl}_{3} / \mathrm{CH}_{3} \mathrm{CN}$ $9: 1(\mathrm{v} / \mathrm{v})$. The absorption spectrum of $\mathbf{1}^{\mathbf{4}+}$ shows an intense band in the near UV $\left(\lambda_{\max }=255 \mathrm{~nm}, \varepsilon_{\max }=51500 \mathrm{M}^{-1} \mathrm{~cm}^{-1}\right)$ and no luminescence, in line with the behaviour of $1,1^{\prime}$-dibenzyl-4,4'-bipyridinium. ${ }^{24}$

A $1.5 \mu \mathrm{M}$ solution of CdSe QDs was titrated with $\mathbf{1}^{\mathbf{4 +}}$ in $\mathrm{CHCl}_{3} /$ $\mathrm{CH}_{3} \mathrm{CN} 9: 1(\mathrm{v} / \mathrm{v})$ and the QD emission at $578 \mathrm{~nm}\left(\lambda_{\text {exc }}=500\right.$ $\mathrm{nm}$ ) was monitored as a function of the concentration of $1^{4+}$. As shown in Fig. 1a, the addition of $\mathbf{1}^{\mathbf{4}+}$ causes a strong quenching of the QD luminescence. Such an emission decrease cannot be ascribed to dynamic quenching, because of the relatively short lifetime of the nanocrystal emission and the very small
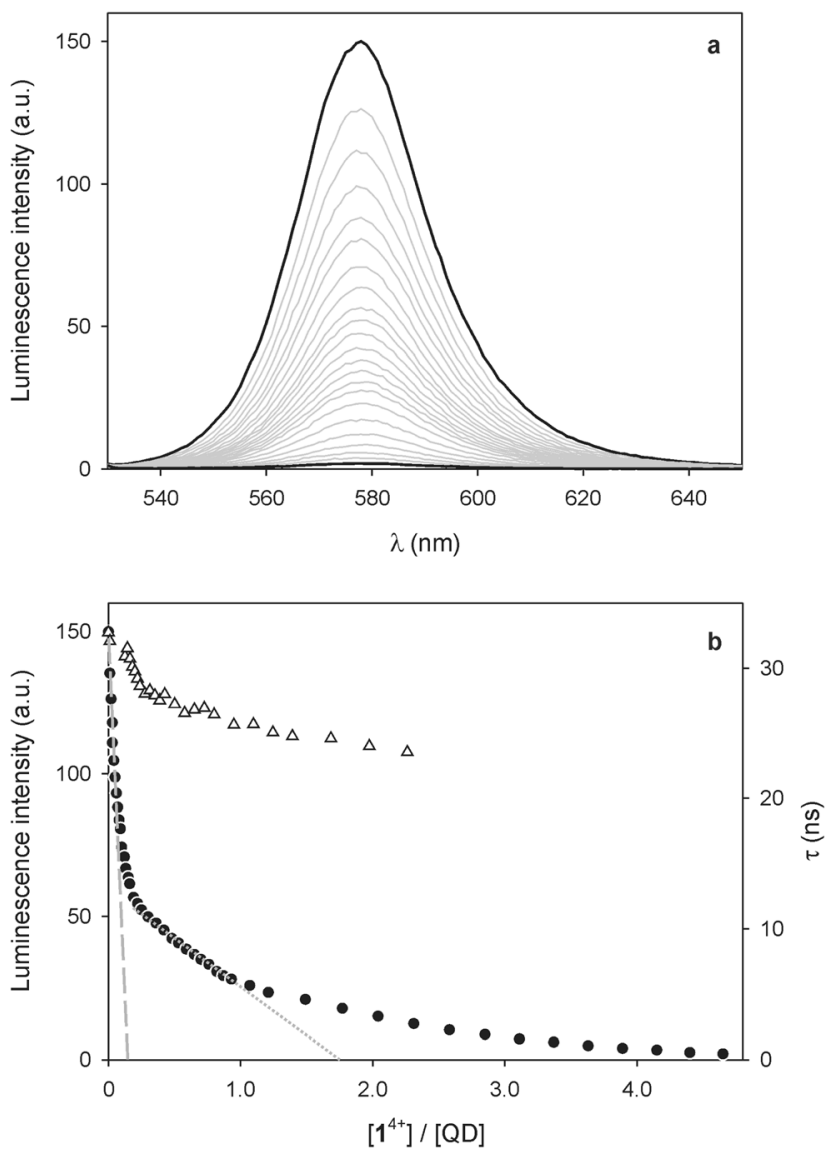

Fig. 1 (a) Luminescence spectral changes observed upon titration of $1.5 \mu \mathrm{M}$ CdSe QDs with $1^{4+}$. (b) Filled circles, left scale: titration plot obtained from the QD luminescence intensity at $578 \mathrm{~nm}$ in the presence of increasing amounts of $1^{4+}$. The dashed and dotted lines show the tangent to the curve at two different slopes and are a guide for the eye, not a fit. Empty triangles, right scale: titration plot obtained from the luminescence lifetime values corresponding to the CdSe emission. Conditions: air equilibrated $\mathrm{CHCl}_{3} / \mathrm{CH}_{3} \mathrm{CN} 9: 1$, room temperature, $\lambda_{\text {exc }}=500 \mathrm{~nm}$. 
concentration of the bipyridinium quencher. Even assuming that dynamic quenching is diffusion controlled $\left(k_{\mathrm{d}}=1.2 \times 10^{10}\right.$ $\left.M^{-1} \mathrm{~s}^{-1}\right),{ }^{25}$ the contribution of dynamic quenching to the emission decrease at the highest employed concentration of $\mathbf{1}^{\mathbf{4}}$ would be less than $0.2 \%$. Moreover, the luminescence lifetime of the QDs is just slightly affected by the addition of the quencher (Fig. 1b). All these findings suggest that the quenching is static, that is, the nanocrystals and $\mathbf{1}^{\mathbf{4 +}}$ are associated in the ground state, as previously observed for related systems., ${ }^{7,9}$

No evidence for ground-state association, however, could be found in the absorption spectra, as the addition of $\mathbf{1}^{\mathbf{4 +}}$ causes no substantial changes in the absorption features of the QDs (Fig. 2a). Nevertheless, a slight increase in absorbance at around $400 \mathrm{~nm}$ and between 500 and $700 \mathrm{~nm}$ is observed; the differential spectrum (Fig. 2b) shows the appearance of new bands with $\lambda_{\max }=401$ and $612 \mathrm{~nm}$, which can be unequivocally ascribed to the presence of bipyridinium radical cations. ${ }^{24}$

As these spectral changes are not observed if the $\mathrm{QD} / \mathbf{1}^{\mathbf{4 +}}$ solution is kept in the dark, reduction of the dicationic
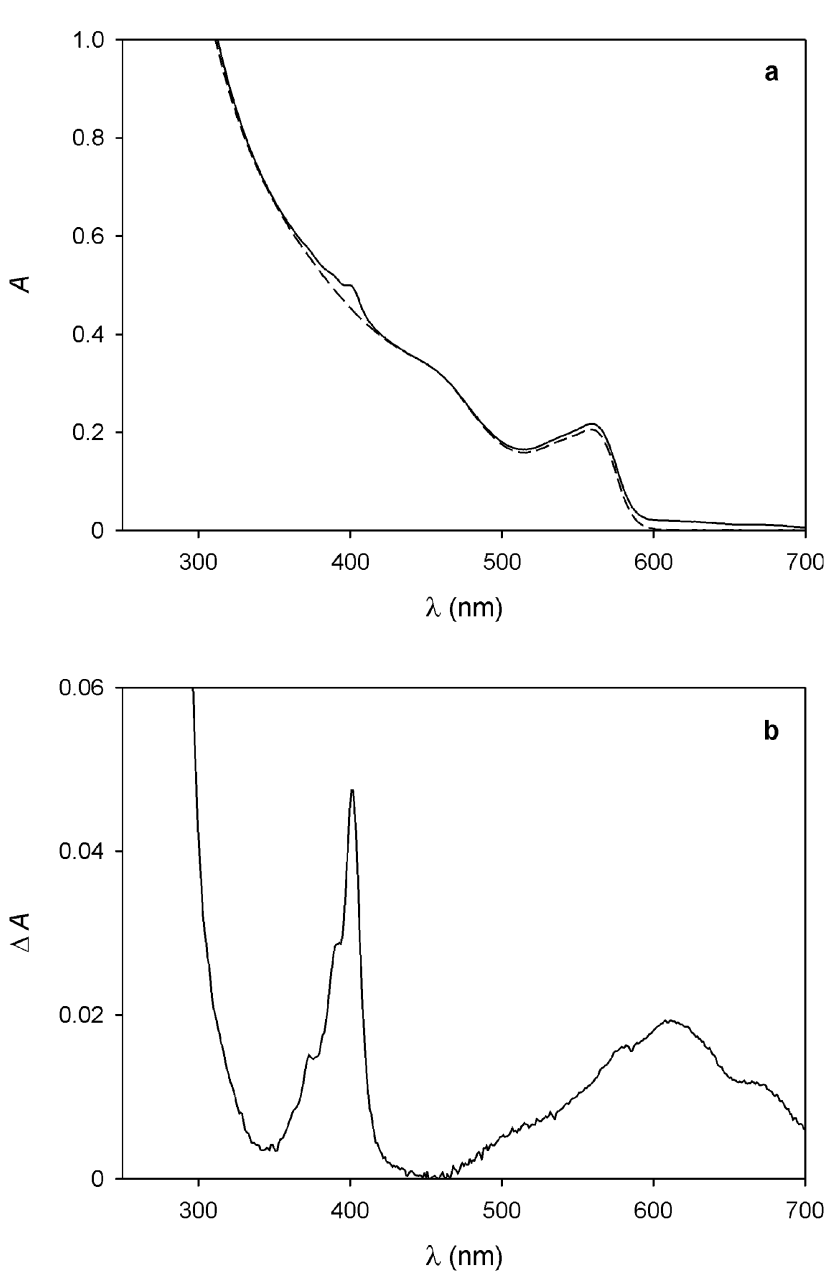

Fig. 2 (a) Absorption spectrum of a $1.5 \mu \mathrm{M}$ solution of CdSe QDs before (full line) and after the spectrofluorimetric titration with 4.6 equivalents of $1^{4+}$ as described in Fig. 1. (b) Difference absorption spectrum of the traces shown in (a), indicating the formation of bipyridinium radical cations. Conditions: air equilibrated $\mathrm{CHCl}_{3}$ / $\mathrm{CH}_{3} \mathrm{CN} 9: 1$, room temperature, $\lambda_{\text {exc }}=500 \mathrm{~nm}$. bipyridinium units of $\mathbf{1}^{\mathbf{4 +}}$ to the corresponding radical cations has to be caused by the light excitation employed in the spectrofluorimetric experiments. Indeed, the photoinduced generation of bipyridinium radical cations was obtained upon visible irradiation of CdSe QD-bipyridinium solutions in the presence of a sacrificial reductant, confirming that the QD luminescence quenching can be ascribed to oxidative electron transfer. ${ }^{\mathbf{1 1 , 1 2}}$ In the present case, it is likely that small amounts of adventitious species (e.g., water) scavenge the photogenerated hole left in the QD valence band, thereby preventing the back electron transfer from the reduced bipyridinium unit to the oxidized nanocrystal. In any instance, the absorption bands of the bipyridinium radical cation disappear after a few minutes in air equilibrated solution, due to slow reoxidation by dioxygen.

In addition to charge transfer, other possible quenching mechanisms in nanocrystal-chromophore complexes are energy transfer (FRET) and formation of non-radiative surface states arising from changes in the capping monolayer. ${ }^{26}$ FRET quenching can be ruled out in the present case because $\mathbf{1}^{\mathbf{4 +}}$ cannot act as an energy acceptor for the CdSe QDs. On the other hand, $\mathbf{1}^{\mathbf{4 +}}$ does not possess functional groups capable of binding to the CdSe surface, and control experiments show that the $\mathrm{PF}_{6}{ }^{-}$counterions have no effect on the QD emission. Moreover, the luminescence quenching occurs immediately after the addition of $\mathbf{1}^{\mathbf{4 +}}$ and is constant thereafter, whereas ligand exchange typically occurs on the time scale of minutes. ${ }^{26}$ The absence of kinetic effects and the reversibility of the quenching observed upon addition of the competitive calixarene host (see below) are consistent with the non-covalent nature of the nanocrystal-quencher association.

Insightful information on the interaction between the QDs and $\mathbf{1}^{\mathbf{4 +}}$ can be gathered from a careful examination of the titration curve (Fig. 1b). At least three different trends for the intensity decrease can be identified, depending on the $1^{\mathbf{4}} / \mathrm{QD}$ ratio. It can be noticed that the tangent to the initial part of the curve (dashed line in Fig. 1b) intercepts the $x$-axis - corresponding to zero emission intensity - at about 0.25 equivalents of $\mathbf{1}^{4^{+}}$. This observation suggests that at low $\mathbf{1}^{\mathbf{4}} / \mathrm{QD}$ ratios one molecule of $\mathbf{1}^{\mathbf{4}}$ (i) can associate with up to four nanocrystals, and (ii) it is able to completely quench the luminescence of any of them.

Because of its size and shape in comparison with those of the quantum dots, it is difficult to imagine that $\mathbf{1}^{\mathbf{4}+}$ can directly link together more than two nanocrystals. It may be hypothesized, however, that such QD pairs could exhibit a stronger ability to bind other nanoparticles than individual QDs, because of the larger surface available for non-covalent interparticle interactions. ${ }^{14}$

On increasing the number of $\mathbf{1}^{\mathbf{4 +}}$ equivalents, non-emissive adducts in which one QD is complexed with two molecules of $\mathbf{1}^{\mathbf{4 +}}$ are also formed (dotted line in Fig. 1b). Not surprisingly, complexes with higher $\mathbf{1}^{\mathbf{4}}$ : QD stoichiometries are obtained as the titration goes on.

\section{CdSe-ZnS core-shell nanocrystals}

CdSe-ZnS core-shell QDs are more interesting for technological applications than bare CdSe nanocrystals, because they possess 
improved stability and photoluminescence efficiency. ${ }^{27}$ Hence, we studied the effect of the bis(bipyridinium) species $\mathbf{1}^{\mathbf{4 +}}$ on QDs composed of a CdSe core $(d=3.8 \mathrm{~nm})$ coated with a $\mathrm{ZnS}$ shell $1.2 \mathrm{~nm}$ thick. These nanocrystal show the typical lowenergy absorption $\left(\lambda_{\max }=579 \mathrm{~nm}, \varepsilon_{\max }=200000 \mathrm{M}^{-1} \mathrm{~cm}^{-1}\right)^{23}$ and emission $\left(\lambda_{\max }=608 \mathrm{~nm}\right.$, fwhm $\left.=39 \mathrm{~nm}\right)$ peaks of their CdSe core.

The addition of $\mathbf{1}^{4+}$ to a $\mathrm{CHCl}_{3} / \mathrm{CH}_{3} \mathrm{CN} 9: 1$ solution of the CdSe-ZnS QDs caused a quenching of the emission (Fig. 3a), that is, a qualitatively similar result to that observed for the CdSe nanocrystals. The shape of the titration curve, however (Fig. 3b), is different from that shown in Fig. 1b. In fact, the emission decrease is much less steep than in the case of the CdSe QDs and a full luminescence quenching is observed after addition of a significantly larger number of $\mathbf{1}^{\mathbf{4}}$ equivalents. The initial part of the curve, whose tangent intercepts the $x$-axis at about 2 equivalents, indicates that at least two $1^{4+}$ molecules are required to completely quench the luminescence of one QD.
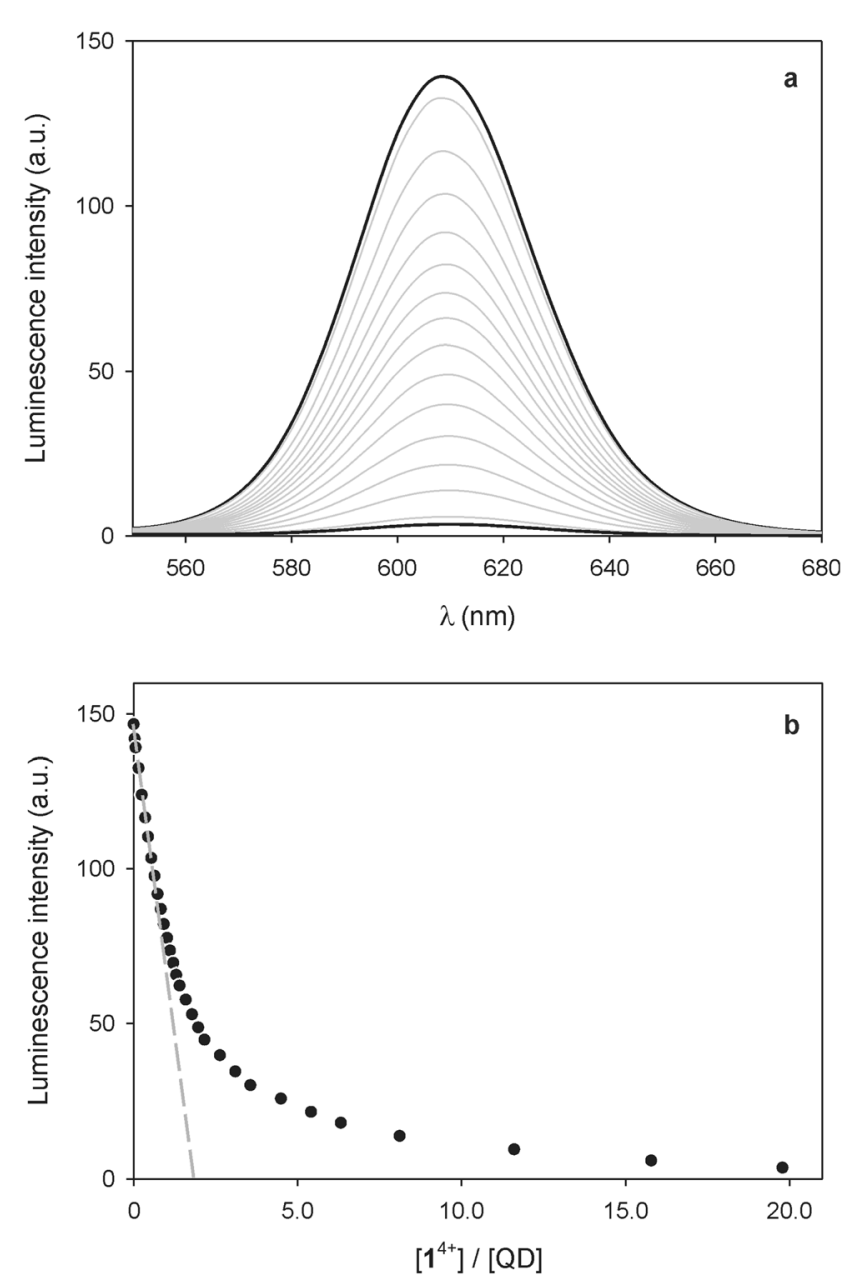

Fig. 3 (a) Luminescence spectral changes observed upon titration of $0.47 \mu \mathrm{M}$ CdSe-ZnS QDs with $1^{4+}$. (b) Titration plot obtained from the QD luminescence intensity at $608 \mathrm{~nm}$ in the presence of increasing amounts of $1^{4+}$. The dashed line shows the tangent to the first part of the curve (it is a guide for the eye, not a fit). Conditions: air equilibrated $\mathrm{CHCl}_{3} / \mathrm{CH}_{3} \mathrm{CN} 9$ : 1, room temperature, $\lambda_{\text {exc }}=500 \mathrm{~nm}$.
This result contrasts with that obtained for the bare CdSe QDs and can be rationalized on the basis of the different size and structure of the CdSe-ZnS QDs. On the one hand, aggregates in which several QDs are bridged by one $\mathbf{1}^{\mathbf{4 +}}$ molecule are unlikely to form because of the larger size of the core-shell particles. On the other hand, the presence of the ZnS shell insulates the CdSe core from the external quencher, thus slowing down the photoinduced electron-transfer process at the basis of quenching. This observation is confirmed by the fact that the absorption bands of the bipyridinium radical cations, observed for the CdSe QDs as a consequence of photoexcitation in the spectrofluorimetric titration, were not detected during the same experiments in the case of the CdSe-ZnS QDs. Photoreduction of the bipyridinium units of $\mathbf{1}^{\mathbf{4}}$ was obtained, though, upon illumination of the CdSe-ZnS QDs with high-intensity visible light $(\lambda>$ $495 \mathrm{~nm}$ ) in the presence of 24 equivalents of $\mathbf{1}^{\mathbf{4}}$. From the intensity of the absorption band at $401 \mathrm{~nm}$ we estimated that, under these conditions, irradiation for $5 \mathrm{~min}$ in deoxygenated solution affords $c a$. $38 \%$ reduction of the bipyridinium units. $\dagger$

\section{QD aggregation triggered by $\mathbf{1}^{4+}$}

To investigate the ability of the bis(bipyridinium) compound $\mathbf{1}^{\mathbf{4}+}$ to non-covalently link together the nanocrystals we performed dynamic light scattering (DLS) and transmission electron microscopy (TEM) experiments on CdSe QD solutions with and without the presence of $\mathbf{1}^{\mathbf{4 +}}$. The CdSe QDs described in the previous section could not be utilized in DLS experiments because they absorb and emit light at the wavelength $(633 \mathrm{~nm})$ used by the instrument to detect light scattering. Hence, the DLS and TEM measurements were performed on smaller CdSe QDs, exhibiting the first exciton absorption peak at $\lambda_{\max }=517 \mathrm{~nm}$, corresponding to a core diameter of $2.5 \mathrm{~nm} .^{23}$ The hydrodynamic diameter of the nanocrystals (Fig. 4a) resulted to be $4.8 \pm 0.1 \mathrm{~nm}$, a figure which is consistent with the core diameter estimated from spectroscopic data and the thickness of the capping ligand shell. ${ }^{28}$ Indeed, the addition of 0.5 equivalents of $\mathbf{1}^{\mathbf{4}+}$ to the QD solution caused a growth of the mean hydrodynamic diameter of the particles to $8.1 \pm 0.7 \mathrm{~nm}$ as well as an increase in their polydispersity (Fig. 4b). This observation is in full agreement with the formation of aggregates consisting of 2 to 4 nanocrystals, as inferred from luminescence titration data.

Further support to the QD aggregation induced by $\mathbf{1}^{\mathbf{4 +}}$ came from TEM experiments. $\uparrow$ The images recorded on substrates obtained from deposition of a dilute solution of the CdSe QDs showed mainly the presence of individual particles in regions with both higher (Fig. 5a) and lower (Fig. 5b) populations of nanocrystals. When 1 equivalent of $\mathbf{1}^{\mathbf{4 +}}$ was added to the QD solution prior to deposition, single nanocrystals could still be detected (Fig. 5c) but small clusters were also found (Fig. 5d). In the presence of 4 equivalents of $\mathbf{1}^{\mathbf{4}+}$ the QDs occurred only in the form of large aggregates and no isolated nanocrystals could be observed (Fig. 5e and f). 

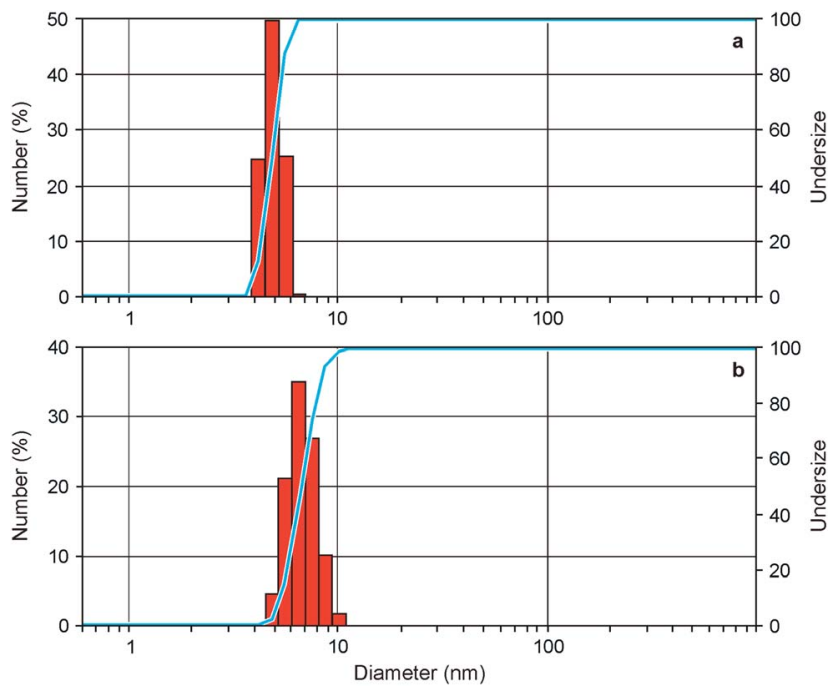

Fig. 4 Differential (red bars) and cumulative (blue line) number distributions of particle size obtained from DLS measurements for the CdSe QDs (a) alone and (b) in the presence of 0.5 equivalents of $1^{4+}$ Conditions: air equilibrated $\mathrm{CHCl}_{3} / \mathrm{CH}_{3} \mathrm{CN} 9: 1$, room temperature.

\section{Luminescence recovery by supramolecular encapsulation of $1^{4+}$}

As pointed out in the introduction, we showed earlier ${ }^{9}$ that QD-bipyridinium adducts could be disrupted by complexing
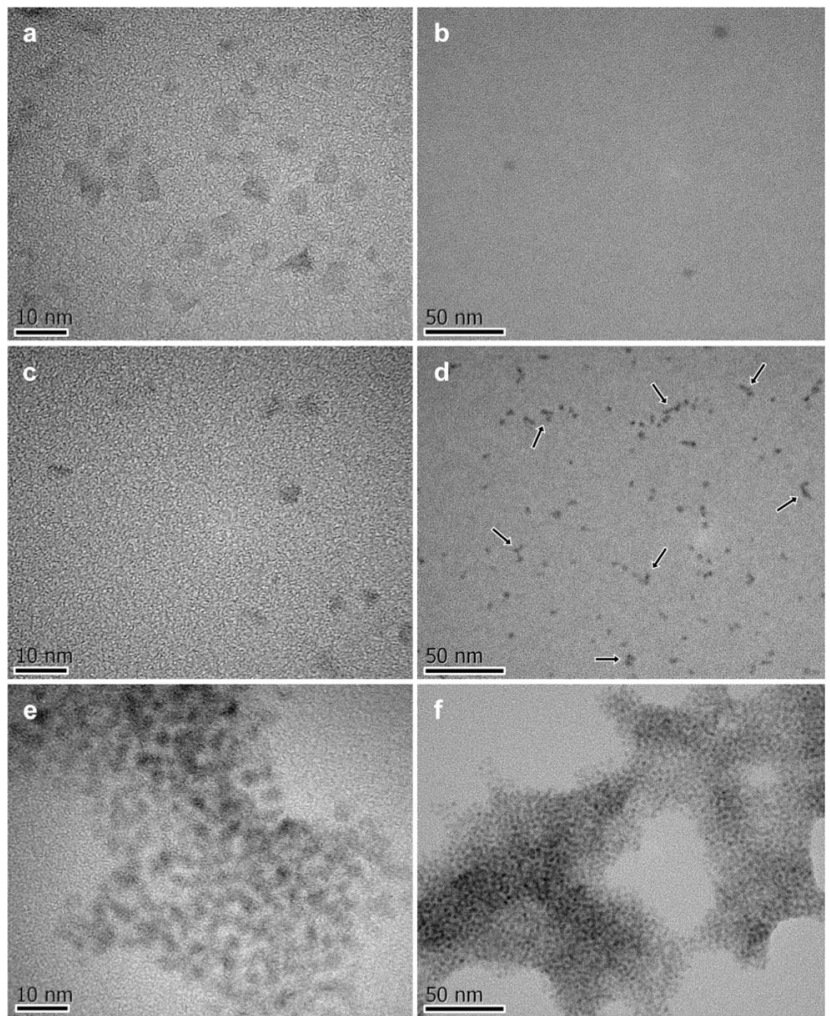

Fig. 5 TEM Images recorded on substrates obtained from deposition of dilute solutions of ( $a$ and b) CdSe QDs, ( $c$ and d) CdSe QDs and $1^{4+}$ in a $1: 1$ ratio, and (e and f) CdSe QDs and $1^{4+}$ in a $1: 4$ ratio. Arrows in (d) indicate small QD aggregates. Scale bar is $10 \mathrm{~nm}$ for (a, c and e) and 50 $n m$ for (b, $d$ and $f)$. the bipyridinium species with the calix[6] arene derivative 2, which can efficiently encapsulate bipyridinium-type guests in organic solution. ${ }^{18,29}$ Hence, we investigated whether the addition of 2 could have an effect on the interaction of $\mathbf{1}^{\mathbf{4 +}}$ with the luminescent nanocrystals.

First of all we performed spectrophotometric titrations to determine the nature and stability of the complex between the bis(bipyridinium) guest and the calixarene host. Upon addition of 2 to $\mathbf{1}^{\mathbf{4}+}$ in $\mathrm{CHCl}_{3} / \mathrm{CH}_{3} \mathrm{CN}$ 9:1 an absorption increase in the 450-550 $\mathrm{nm}$ region, typical of the inclusion of a bipyridinium unit within the cavity of $2{ }^{28}$ was observed (Fig. 6a). The corresponding titration curve obtained from the absorbance values at $460 \mathrm{~nm}$ (Fig. 6b) could be satisfactorily fitted with a binding model implying the formation of two adducts (eqn (1) and (2)); ${ }^{30}$ namely, a $1: 1$ complex in which one molecule of 2 encircles a bipyridinium unit of $\mathbf{1}^{4+}\left(K_{1: 1}=1.3 \times 10^{5} \mathrm{M}^{-1}\right)$ and a $2: 1$ complex in which two calixarene molecules surround both bipyridinium units of one $\mathbf{1}^{\mathbf{4 +}}$ species $\left(K_{2: 1}=2.5 \times 10^{3} \mathrm{M}^{-1}\right)$.
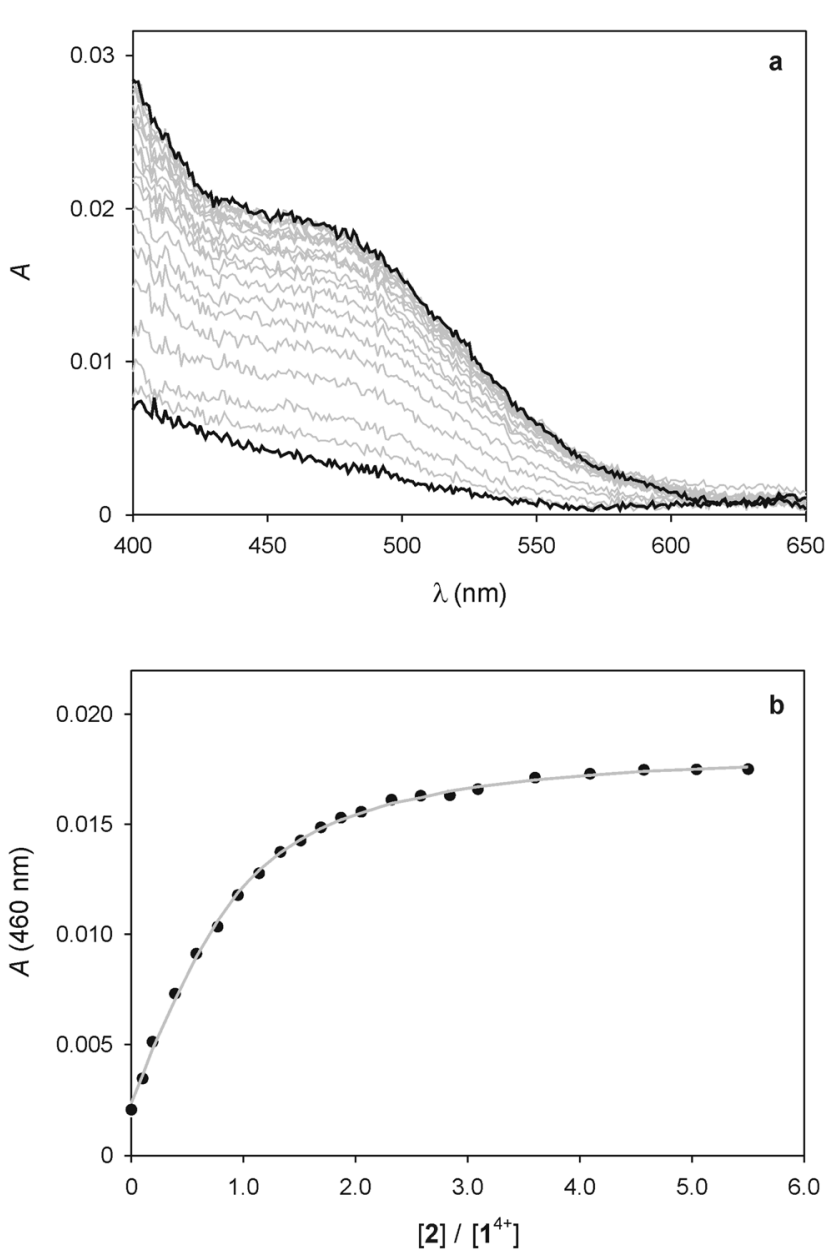

Fig. 6 (a) Absorption spectral changes observed upon titration of 25 $\mu \mathrm{M} 1^{4+}$ with 2. (b) Titration plot obtained from the absorption values at $460 \mathrm{~nm}$. The grey curve is the data fitting according to the $1: 1$ and $2: 1$ binding model described in the text (eqn (1) and (2)). Conditions: air equilibrated $\mathrm{CHCl}_{3} / \mathrm{CH}_{3} \mathrm{CN} 9: 1$, room temperature. 
The value of $K_{1: 1}$ is in line with those found in previous investigations for the $1: 1$ complex between 2 and 1,1'-dialkyl-4,4'-bipyridinium guests. On the other hand, the significantly lower value of $K_{2}: 1$ indicates that the presence of a first calixarene unit around $\mathbf{1}^{\mathbf{4}+}$ hampers the association of a second molecule of 2 . This finding is not unexpected if one considers that the distance between the two bipyridinium units of $\mathbf{1}^{\mathbf{4 +}}$ is short in relation to the length of 2 , resulting in a mutual steric hindrance of the two calixarene hosts in the $2: 1$ complex.

Variable amounts of calixarene 2 were added to assemblies of $\mathbf{1}^{4+}$ and either CdSe $\left(\lambda_{\text {max,abs }}=560 \mathrm{~nm}, d=3.3 \mathrm{~nm}\right)$ or CdSe$\mathrm{ZnS}$ QDs in $\mathrm{CHCl}_{3} / \mathrm{CH}_{3} \mathrm{CN}$ 9:1 and spectrofluorimetric measurements were carried out on the resulting solutions. Upon addition of 5 equivalents of 2 (with respect to $\mathbf{1}^{\mathbf{4}}$ ) to a solution containing CdSe QDs $(1.5 \mu \mathrm{M})$ and ${1^{4+}}^{+}$in a 1 : 4.6 ratio, only less than $1 \%$ of the initial emission intensity was restored. Similarly, the addition of 38 equivalents of 2 (with respect to $1^{\mathbf{4 +}}$ ) to a solution containing CdSe-ZnS QDs $(0.47 \mu \mathrm{M})$ and $\mathbf{1}^{\mathbf{4}}$ in a 1 : 20 ratio caused $<1 \%$ recovery of the luminescence. Notably, the emission of CdSe-ZnS QDs $(0.43 \mu \mathrm{M})$ and 1,1'-dibenzyl-4,4'bipyridinium in a $1: 19$ ratio was fully restored after the addition of 20 equivalents of 2 (with respect to the bipyridinium guest). ${ }^{9}$ The comparison of these results suggests that the binding of the bis(bipyridinium) $\mathbf{1}^{\mathbf{4}+}$ species to the nanocrystals is stronger than that of a structurally related compound bearing a single bipyridinium unit. The larger size of $\mathbf{1}^{\mathbf{4 +}}$, its twice as large electric charge, and its lower affinity for chloroform may be among the reasons for such a behaviour. It may also be considered that complexation of $\mathbf{1}^{\mathbf{4}}$ by 2 could be hampered by aggregation of the nanocrystals triggered by the former.

$$
\begin{gathered}
\mathbf{1}^{4+}+\mathbf{2} \stackrel{K_{1: 1}}{\rightleftarrows}[1 \subset \mathbf{2}]^{4+} \\
{[1 \subset \mathbf{1}]^{4+}+\mathbf{2} \stackrel{K_{2: 1}}{\rightleftarrows}\left[1 \subset(2)_{2}\right]^{4+}}
\end{gathered}
$$

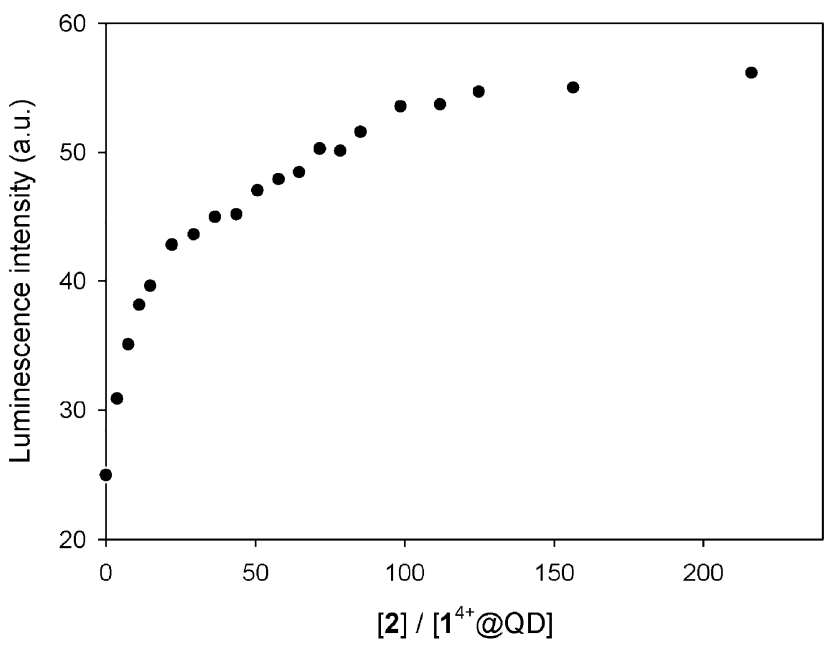

Fig. 7 Luminescence spectral changes at $578 \mathrm{~nm}$ observed upon titration of a $1: 1$ mixture of CdSe QDs and $1^{4+}(1.5 \mu \mathrm{M})$ with the calixarene host 2. Conditions: air equilibrated $\mathrm{CHCl}_{3} / \mathrm{CH}_{3} \mathrm{CN} 9$ : 1, room temperature, $\lambda_{\text {exc }}=500 \mathrm{~nm}$.
Nevertheless, the titration of a $1.5 \mu \mathrm{M} 1: 1$ mixture of CdSe QDs and $\mathbf{1}^{\mathbf{4}}$ (in which $\mathrm{ca}$. $87 \%$ of the nanocrystals luminescence is quenched, see Fig. 1b) with 2 shows a recovery of up to $30 \%$ of the original emission intensity of the QDs after the addition of a large excess (215 equivalents) of the calixarene host (Fig. 7). Conversely, the addition of the same excess of 2 to the QDs (in the absence of $\mathbf{1}^{\mathbf{4}}$ ) caused a $8 \%$ emission decrease. These observations indicate that complexation of $\mathbf{1}^{4+}$ by 2 causes the disassembly of the non-luminescent adducts consisting of the QDs and the bis(bipyridinium) species.

\section{Conclusions}

We have investigated the interaction of CdSe and CdSe-ZnS luminescent nanocrystals with the ditopic bis(bipyridinium) species $\mathbf{1}^{\mathbf{4}+}$ in organic solution. Our results show that the nanocrystal and molecular components undergo association in the ground state, yielding complexes in which the luminescence of the quantum dots is strongly quenched. Interestingly, $\mathbf{1}^{\mathbf{4 +}}$ can associate and quench as many as four CdSe nanocrystals, possibly owing to cooperative interparticle binding phenomena. The ability of $\mathbf{1}^{\mathbf{4 +}}$ to promote aggregation of the quantum dots is confirmed by DLS and TEM experiments.

Visible light irradiation of the adducts causes the formation of bipyridinium radical cations, confirming that the luminescence quenching mechanism is an electron-transfer process from the excited electron in the conduction band of the nanocrystal to the LUMO of the bipyridinium quencher. The presence of a ZnS shell around the CdSe core does not prevent the electron-transfer quenching process but it does reduce its efficiency.

Calix[6]arene 2, which is able to encapsulate both bipyridinium units of $\mathbf{1}^{\mathbf{4 +}}$ by virtue of supramolecular interactions, was used to promote the decomplexation of $\mathbf{1}^{\mathbf{4 +}}$ from the quantum dots. Indeed, the addition of an excess of 2 to a solution containing the nanocrystal $/ \mathbf{1}^{\mathbf{4}}$ complexes causes a recovery of the luminescence of the quantum dots, indicating the partial disassembly of the complexes.

These results demonstrate that supramolecular interactions are a convenient way to control the aggregation of semiconductor nanocrystals. The combination of molecular recognition and self-assembly concepts with luminescent quantum dots is a promising route for the generation of nanostructured arrays with controlled morphologies and properties. Such a task is important for improving the performances of quantum dotbased optoelectronic devices.

\section{Acknowledgements}

This work was supported by the European Union (EU) (Hysens Project, grant no. 263091, ATOMIN project, grant no. POIG.02.01.00-12-023/08), the Ministero dell'Università e della Ricerca (PRIN 2010CX2TLM) and the University of Bologna. M. O. thanks the National Science Centre (grant no. UMO 2011/01/ N/ST5/02550) and the Foundation for Polish Science for a MPD programme fellowship co-financed by the EU European Regional Development Fund. K. S. acknowledges financial 
support from the National Science Centre (grant no. UMO-2011/ 03/B/ST5/01495).

\section{Notes and references}

1 Semiconductor nanocrystal quantum dots, ed. A. L. Rogach, Springer-Verlag, Wien, Austria, 2008.

2 A. M. Smith and S. Nie, Acc. Chem. Res., 2010, 43, 190.

3 U. Resch-Genger, M. Grabolle, S. Cavaliere-Jaricot, R. Nitschke and T. Nann, Nat. Methods, 2008, 5, 763.

4 Quantum dot sensors: technology and commercial applications, ed. J. F. Callan and F. M. Raymo, Pan Stanford Publishing, Singapore, 2013.

5 I. Yildiz, E. Deniz and F. M. Raymo, Chem. Soc. Rev., 2009, 38, 1859; I. L. Medintz and H. Mattoussi, Phys. Chem. Chem. Phys., 2009, 11, 17; N. Hildebrandt, ACS Nano, 2011, 5, 5286; T. L. Doane and C. Burda, Chem. Soc. Rev., 2012, 41, 2885; R. Freeman and I. Willner, Chem. Soc. Rev., 2012, 41, 4067; R. Freeman, J. Girsh and I. Willner, ACS Appl. Mater. Interface, 2013, 5, 2815; T. Avellini, C. Lincheneau, F. Vera, S. Silvi and A. Credi, Coord. Chem. Rev., 2014, 263-264, 151. 6 See, e.g.: Y. Chen, R. Thakar and P. T. Snee, J. Am. Chem. Soc., 2008, 130, 3744; I. L. Medintz, M. H. Stewart, S. A. Trammell, K. Susumu, J. B. Delehanty, B. C. Mei, J. S. Melinger, J. B. Blanco-Canosa, P. E. Dawson and H. Mattoussi, Nat. Mater., 2010, 9, 676; Z. Erno, I. Yildiz, B. Gorodetsky, F. M. Raymo and N. R. Branda, Photochem. Photobiol. Sci., 2010, 9, 249; M. Amelia, A. Lavie-Cambot, N. D. McClenaghan and A. Credi, Chem. Commun., 2011, 47, 325; W. R. Algar, D. Wegner, A. L. Huston, J. B. BlancoCanosa, M. H. Stewart, A. Armstrong, P. E. Dawson, N. Hildebrandt and I. L. Medintz, J. Am. Chem. Soc., 2012, 134, 1876; S. Impellizzeri, B. McCaughan, J. F. Callan and F. M. Raymo, J. Am. Chem. Soc., 2012, 134, 2276; R. Freeman, T. Finder, L. Bahshi, R. Gill and I. Willner, Adv. Mater., 2012, 24, 6416; J. Aguilera-Sigalat, V. F. Pais, A. Domenech-Carbo, U. Pischel, R. E. Galian and J. PerezPrieto, J. Phys. Chem. C, 2013, 117, 7365.

7 M. Amelia, M. Font and A. Credi, Dalton Trans., 2011, 40, 12083; M. Amelia and A. Credi, Inorg. Chim. Acta, 2012, 381, 247.

8 A. Boulesbaa, A. Issac, D. Stockwell, Z. Huang, J. Huang, J. Guo and T. Lian, J. Am. Chem. Soc., 2007, 129, 15132; S.-C. Cui, T. Tachikawa, M. Fujitsuka and T. Majima, J. Phys. Chem. C, 2011, 115, 1824; J. Aguilera-Sigalat, J. M. Casas-Solvas, M. C. Morant-Minana, A. VargasBerenguel, R. E. Galian and J. Perez-Prieto, Chem. Commun., 2012, 48, 2573; A. Iagatti, R. Flamini, M. Nocchetti and L. Latterini, J. Phys. Chem. C, 2013, 117, 23996.

9 B. Gadenne, I. Yildiz, M. Amelia, F. Ciesa, A. Secchi, A. Arduini, A. Credi and F. M. Raymo, J. Mater. Chem., 2008, 17, 2022.

$10 \mathrm{~J}$. Bebie, M. A. A. Schoonen, M. Fuhrmann and D. R. Strongin, Geochim. Cosmochim. Acta, 1998, 62, 633; M. Tiemann, F. Marlow, F. Brieler and M. Lindén, J. Phys. Chem. B, 2006, 110, 23142; M. Wang, Q. Zhang, W. Hao and Z.-X. Sun, Chem. Cent. J., 2011, 5, 73; J. Saikiaa, B. Sahab and G. Das, J. Colloid Interface Sci., 2014, 416, 235.

11 I. Yildiz and F. M. Raymo, J. Mater. Chem., 2006, 16, 1118; I. Yildiz, M. Tomasulo and F. M. Raymo, Proc. Natl. Acad. Sci. U. S. A., 2006, 103, 11457.

12 S. Logunov, T. Green, S. Marguet and M. A. El-Sayed, J. Phys. Chem. A, 1998, 102, 5652; C. Burda, T. C. Green, S. Link and M. A. El-Sayed, J. Phys. Chem. B, 1999, 103, 1783.

13 M. Grzelczak, J. Vermant, E. M. Furst and L. M. Liz-Marzán, ACS Nano, 2010, 4, 3591; R. Schreiber, J. Do, E.-M. Roller, T. Zhang, V. J. Schüller, P. C. Nickels, J. Feldmann and T. Liedl, Nat. Nanotechnol., 2014, 9, 74.

14 T. Wang, D. LaMontagne, J. Lynch, J. Zhuang and Y. C. Cao, Chem. Soc. Rev., 2013, 42, 2804.

15 H. Zhang, E. W. Edwards, D. Y. Wang and H. Möhwald, Phys. Chem. Chem. Phys., 2006, 8, 3288; Y. Gao and Z. Tang, Small, 2011, 7, 2133; C. Zhang, R. J. Macfarlane, K. L. Young, C. H. J. Choi, L. Hao, E. Auyeung, G. Liu, X. Zhou and C. A. Mirkin, Nat. Mater., 2013, 12, 741.

16 D. Vanmaekelbergh and P. Liljeroth, Chem. Soc. Rev., 2005, 34, 299; D. V. Talapin, J. S. Lee, M. V. Kovalenko and E. V. Shevchenko, Chem. Rev., 2010, 110, 389.

17 R. J. Alvarado, J. Mukherjee, E. J. Pacsial, D. Alexander and F. M. Raymo, J. Phys. Chem. B, 2005, 109, 6164.

18 A. Credi, S. Dumas, S. Silvi, M. Venturi, A. Arduini, A. Pochini and A. Secchi, J. Org. Chem., 2004, 69, 5881.

19 A. Credi and L. Prodi, J. Mol. Struct., 2014, DOI: 10.1016/ j.molstruc.2014.03.028, in press.

20 Z. A. Peng and X. Peng, J. Am. Chem. Soc., 2001, 123, 183; J. Li, Y. A. Wang, W. Guo, J. C. Keay, T. D. Mishima, M. B. Johnson and X. Peng, J. Am. Chem. Soc., 2003, 125, 12567.

21 Z. A. Peng and X. Peng, J. Am. Chem. Soc., 2002, 124, 3343; C. R. Bullen and P. Mulvaney, Nano Lett., 2004, 4, 2303.

22 D. Chen, F. Zhao, H. Qi, M. Rutherford and X. Peng, Chem. Mater., 2010, 22, 1437.

23 W. W. Yu, L. Qu, W. Guo and X. Peng, Chem. Mater., 2003, 15, 2854.

24 R. Ballardini, A. Credi, M. T. Gandolfi, C. Giansante, G. Marconi, S. Silvi and M. Venturi, Inorg. Chim. Acta, 2007, 360, 1072.

25 M. Montalti, A. Credi, L. Prodi and M. T. Gandolfi, Handbook of photochemistry, CRC Press, Boca Raton, 3rd edn, 2006.

26 T. Blaudeck, E. I. Zenkevich, M. Abdel-Mottaleb, K. Szwaykowska, D. Kowerko, F. Cichos and C. von Borczyskowski, ChemPhysChem, 2012, 13, 959.

27 M. A. Hines and P. Guyot-Sionnest, J. Phys. Chem., 1996, 100, 468.

28 J. M. Tsay, S. Doose and S. Weiss, J. Am. Chem. Soc., 2006, 128, 1639.

29 A. Arduini, R. Bussolati, A. Credi, A. Secchi, S. Silvi, M. Semeraro and M. Venturi, J. Am. Chem. Soc., 2013, 135, 9924; M. Semeraro, A. Secchi, S. Silvi, M. Venturi, A. Arduini and A. Credi, Inorg. Chim. Acta, 2014, 417, 258.

30 R. A. Binstead, SPECFIT, Spectrum Software Associates, Chapel Hill, NC, 1996. 